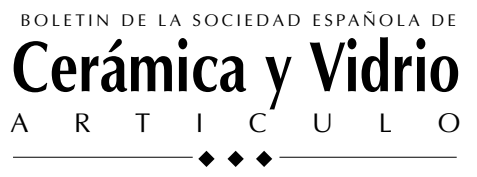

\title{
La innovación tecnológica en la industria cerámica de Castellón
}

\author{
A. ESCARDINO \\ Instituto de Tecnología Cerámica.Asociación de Investigación de las Industrias Cerámicas. Universitat Jaume I. Castellón. España.
}

\begin{abstract}
La innovación tecnológica se produce como consecuencia de la interacción entre los tres términos que componen el segundo miembro del polinomio siguiente: Innovación $=$ (Recursos tecnológicos + Recursos humanos $)+$ Recursos financieros. En la ponencia se analiza el proceso interactivo entre estos tres componentes, en relación con el desarrollo experimentado, durante los últimos años, por la industria española de baldosas cerámicas y la de fritas y colorantes cerámicos, el 95\% de cuya producción está concentrado en un área muy reducida de la provincia de Castellón, constituyendo lo que, en términos económicos, se denomina "un sistema productivo local" (Cluster). Se estudia el papel que han jugado la iniciativa empresarial y la disponibilidad de la tecnología y del personal técnico cualificado necesarios para implantar la innovación industrial que ha permitido el extraordinario crecimiento experimentado, en los últimos diez años, por dichos subsectores industriales. Desarrollo al que ha contribuido la existencia de un entramado socio-económico-institucional que ha actuado como catalizador del proceso interactivo entre dichos factores.
\end{abstract}

\section{Technological innovation in the Castellón ceramic industry}

Technological innovation occurs a a result of the integration of three terms that make up the second member of the following polynomial: innovation $=$ (Technological resources + Human resources). The paper analyses the interactive process amongst these three components in relation to the development witnessed in recent years by the spanish ceramic tile as well as the frit and ceramic colour industry, $95 \%$ of those production is concentrated in a very limited area of Castellon province, forming what is known in economics as a cluster. The paper studies the role of entrepreneurial initiative and the avalability of the technology and qualified technical personnel required in implementing the industrial innovation that has yielded such extraordinary growth of these industrial subsectors in the last ten years. A development fostered by the presence of a socioeconomic-institutional network that has catalysed the interactive process amongst these factors

\section{INTRODUCCIÓN}

La mayoría de las grandes empresas y un número cada vez mayor de pequeñas y medianas empresas (PYMEs) se están convenciendo o están ya convencidas de la necesidad de gestionar lo mejor posible el conjunto de los recursos tecnológicos, humanos, financieros y comerciales en el marco de una estrategia global. Según los expertos en la materia, las empresas que triunfarán en el futuro, serán aquellas que tengan la preocupación permanente de movilizar del mejor modo posible todos estos recursos, en especial los tecnológicos.

El crecimiento y la competitividad de las empresas industriales están y lo estarán cada vez más, en un futuro inmediato, íntimamente ligados a su capacidad para innovar, en los productos y en los procesos, con la rapidez necesaria para poder atender la demanda de los mercados en lo que a calidad, precio e interés de los productos ofrecidos se refiere. Es decir, están condicionados al potencial de las empresas para desarrollar innovación tecnológica.

La innovación tecnológica consiste en "aplicar a escala industrial una nueva tecnología para poner a disposición de la sociedad un producto inédito (de características mejoradas, con menor coste de fabricación, con propiedades nuevas, etc.), un proceso de fabricación novedoso (más sencillo, mejor controlado, con mayor producción, etc.) o cualquier otro beneficio no conocido previamente".

El proceso de innovación tecnológica o industrial se produce como consecuencia de la interacción entre los tres componentes del segundo miembro del siguiente polinomio:
Innovación industrial $=[$ Tecnología inédita (generada o adaptada) + Personal técnico cualificado para su optima aplicación y eventual mejora)] +Iniciativa empresarial(capital).

Esta expresión puede formularse de una forma más general del modo siguiente:

Innovación industrial $=$ (Recursos tecnológicos inéditos + Recursos humanos) + Recursos Financieros (capital).

\subsection{Recursos tecnológicos}

Según definición de la OCDE, la Tecnología es "el conjunto de información y conocimientos sistemáticos necesarios para la fabricación de un producto, para la aplicación de un proceso o para la prestación de un servicio".

La tecnología inédita necesaria para realizar innovación puede ser "generada", mediante la realización de actividades de I+D orientadas a alcanzar un objetivo concreto, o "transferida" , procedente de otros campos de actividad industrial, asimilándola y adaptándola de modo que su puesta en practica en el nuevo contexto constituya una novedad.

\subsubsection{GENERACIÓN DE TECNOLOGÍA}

Para generar la tecnología necesaria para la innovación industrial es preciso aprovechar la información existente en la bibliografía, complementándola con la realización de "investigación básica dirigida" y de "investigación aplicada" orientadas a resolver el problema planteado o a alcanzar el objetivo previamente establecido (obtención de un producto nuevo, 
mejora de la calidad o abaratamiento de un producto existente, mejora del proceso de fabricación o de alguna de sus etapas, reducción del impacto ambiental, etc.).

Una vez finalizada esta etapa experimental, es imprescindible completarla con la fase de "desarrollo tecnológico", es decir con la realización del conjunto de actividades complementarias necesarias para tratar de reproducir, a escala de fabricación, los resultados obtenidos en el laboratorio. Esta etapa puede ser compleja, ya que: a) con frecuencia, las características del equipo que se ha utilizado para realizar el estudio a escala de laboratorio difieren notablemente de las del equipo que se ha de emplear para desarrollar la operación o proceso a escala industrial.; b) se ha de resolver la problemática inherente al cambio de escala de las diferentes etapas del proceso; c) en el caso de que el proceso industrial se desarrolle en régimen continuo y estacionario, dado que la investigación experimental que se realiza a escala de laboratorio se efectúa habitualmente en régimen discontinuo o intermitente, es preciso adaptar los resultados de la misma a las características del equipo industrial que se ha de utilizar.

Al llegar a este punto, es importante poner de manifiesto que los resultados de una investigación bien planificada y correctamente desarrollada que no conduzcan a la obtención de un producto competitivo, en calidad y precio, o a la mejora de un proceso, con la consiguiente repercusión económica, contribuirá a la ampliación del conocimiento pero no resultará útil para realizar innovación. Esta puntualización creo que es muy importante ya que frecuentemente se asocia la investigación con la innovación, sin tener en cuenta que la única investigación cuyos resultados se utilizan para hacer innovación es aquella que, a corto o medio plazo, permite la puesta a punto de una nueva tecnología que simplificará o mejorará un proceso de fabricación o que conducirá a la obtención de un nuevo producto suficientemente competitivo por su calidad y precio (condición importantísima, esta ultima, que no se suele tener en cuenta por la mayoría de los investigadores cuando eligen líneas de trabajo).

Ahora bien, ¿quién genera la tecnología inédita?. La investigación aplicada y el desarrollo tecnológico subsiguiente, necesarios para generar una tecnología nueva, pueden realizarse en la propia empresa, si posee equipo y recursos humanos suficientes, en otra empresa que posteriormente le transfiere la tecnología, en un Organismo Público de Investigación o en un Centro de Innovación y Tecnología, contratándola en cualquiera de estos últimos casos.

\subsubsection{TRANSFERENCIA DE TECNOLOGÍA}

La investigación científica y el ulterior desarrollo de la tecnología resultante de la misma no son el único camino que conduce a la innovación, aún cuando la mentalidad reinante identifica o asocia normalmente la innovación con la investigación.

En efecto, es un hecho contrastado que la transferencia de tecnología, procedente de sectores industriales distintos de aquel al que se desea aplicar, es origen de un número mayor de acciones de innovación que la aplicación de la tecnología generada con investigación propia, cuyo desarrollo es mas lento, más costoso y más aventurado. Este hecho es especialmente aplicable a las PYMEs que, por su tamaño (menos de 250 empleados), no poseen, en la mayor parte de los casos, la infraestructura necesaria para generar, mediante acciones propias de I+D, ni siquiera una pequeña parte de la tecnología que necesitan para innovar a un ritmo tal que les permita mantener o incrementar su competitividad. En general, estas empresas suelen estar más pendientes de la productividad de sus instalaciones que de realizar acciones de I+D. Por otra parte, la baja rentabilidad a corto plazo de los recursos necesarios para llevar a cabo dichas acciones trae consigo que, solo en muy contadas ocasiones, las PYMEs asuman dichas actividades como algo que debe realizarse en la propia empresa. Generalmente se limitan a realizar la innovación industrial asimilando y desarrollando la tecnología adquirida. Tal es el caso de la mayor parte de las empresas del sector cerámico en España.

Las PYMEs japonesas y alemanas utilizan en mayor proporción la transferencia de tecnología que la investigación propia para innovar, porque la consideran más eficaz, en razón de los costes, rapidez de aplicación y probabilidades de éxito, por lo que concentran casi todos sus recursos en la asimilación y desarrollo o aplicación de tecnologías transferidas. Como dice Tom Peters, en su obra "Le Chaos Management", la imitación no tiene porque frenar la imaginación creadora.

¿Cómo se consigue la transferencia de tecnología?. Esta acción se desarrolla habitualmente en tres fases: prospección, asimilación o adaptación e implantación, a escala industrial, de tecnologías empleadas en otros procesos de fabricación o en otras actividades industriales, pero que son inéditas en el proceso o actividad al que se propone aplicarlas. En la fase de prospección se captan las necesidades o deficiencias tecnológicas de una o varias empresas industriales análogas y se trata de descubrir nuevas ideas para tratar de solucionar la problemática detectada, asistiendo para ello a foros de tecnología y a ferias, consultando bases de datos, artículos científicos, etc., relacionados con dicha problemática. Caso de que la nueva tecnología detectada no requiera de una adaptación previa a las peculiaridades del proceso de fabricación al que se va a aplicar, se implanta directamente. Si la nueva tecnología no se puede aplicar directamente al proceso, es preciso realizar una acción previa de desarrollo tecnológico con la finalidad de adaptarla al mismo.

¿Quién efectúa la acción de transferir tecnología a las empresas?. Las tres fases antes descritas pueden ser realizadas, total o parcialmente: a) por personal técnico cualificado perteneciente a la propia empresa (caso de que tenga una plantilla suficientemente amplia y con la preparación adecuada); b) por otra empresa, normalmente de mayor tamaño que, por diferentes razones (ser socio, ser suministradora de materias primas elaboradas, etc.) esté interesada en suministrar a la PYME la tecnología que necesita; c) por un Centro de Innovación y Tecnología especializado en el sector industrial al que pertenece la empresa demandante, contratado al efecto.

\subsubsection{ASISTENCIA TÉCNICA Y SERVICIOS}

La implantación a escala industrial de la tecnología, tanto si es generada como si es transferida, requiere, como se ha indicado anteriormente, la realización de un conjunto de actividades que muchas veces no puede efectuar, en su totalidad, el personal técnico de la PYME que está tratando de innovar. En ese caso, la PYME necesita disponer de un servicio de asistencia técnica externo complementario. Este servicio puede prestarse por personal de la empresa que le ha transferido la tecnología o por personal técnico de un Centro de Innovación y Tecnología especializado.

Además de las actividades que conducen al perfeccionamiento de los procesos de fabricación existentes, a la mejora 
de la calidad y utilidad de los productos que se están fabricando y al descubrimiento y desarrollo de nuevos productos y de técnicas de fabricación inéditas, o sea a la innovación, las PYMEs necesitan desarrollar, por si mismas o con la colaboración de terceros (otra empresa o un Centro de Innovación y Tecnología), otras actividades complementarias para sustentar la innovación implantada, tales como: a) la normalización de los artículos fabricados, en la medida que esta acción se traduzca en beneficios económicos para la empresa; b) detección y solución rápidas de los obstáculos que dificulten inopinadamente la actividad productiva; c) elaboración de ensayos diversos para comprobar la idoneidad de las materias primas empleadas, el normal desarrollo del proceso de fabricación y las propiedades y características de los productos industriales fabricados. Hay muchas PYMEs que no disponen en sus laboratorios del equipamiento suficiente para realizar los análisis y los ensayos que se requieren para efectuar las pruebas de control de calidad precisas o para detectar y establecer la naturaleza de los problemas que pueden obstaculizar imprevistamente el correcto desarrollo del proceso de fabricación. En este caso las empresas necesitan disponer de laboratorios externos, acreditados si es posible oficialmente, de análisis y ensayos, especializados en la problemática del sector industrial en el que se ubican.

\subsection{Recursos humanos $y$ financieros}

Para reforzar lo que se ha dicho anteriormente sobre la investigación y la generación y transferencia de tecnología y como confirmación de la interrelación existente entre los tres componentes que se consideran necesarios para desarrollar la innovación industrial, viene a cuento la cita que se refiere a continuación. En un seminario que se desarrolló en el Escorial, en febrero de 1989, sobre el "Pasado, Presente y Futuro de las relaciones Universidad-Empresa", el Prof. Castells, analizando los factores relacionados con el incremento de productividad de las empresas, sostenía que "una investigación bien realizada que no se pueda aplicar a la producción no contribuirá al desarrollo económico; una utilización masiva de nuevas tecnologías que no coincida con el desarrollo del tejido productivo adecuado no permitirá el pleno aprovechamiento de aquellas, y una investigación, producción y consumo que no cuenten con la disponibilidad de los recursos humanos cualificados que son necesarios para el desarrollo y aplicación de esas nuevas tecnologías en la producción y en la gestión, se verá rápidamente abocada a un cuello de botella".

En estas opiniones se insiste sobre el papel trascendental que juega, en el proceso de innovación (íntimamente ligado al desarrollo económico), la existencia de un entorno empresarial dispuesto a invertir para desarrollar el tejido fabril preciso y disponer de personal técnico cualificado para asimilar, aplicar y mejorar (si procede) la tecnología necesaria.

El papel que juega el capital y la iniciativa empresarial en el proceso de innovación es tan evidente que sobran los comentarios. De nada sirve disponer de nuevas tecnologías y de personal técnico cualificado, si no existe inquietud por innovar y capital para financiar dicho proceso.

Para las PYMEs, la profesionalidad de su equipo humano es, hoy día, mucho más importante que hace unos años. El factor que contribuye mayormente a la excelencia de sus capacidades diferenciadoras es la formación que posee su personal técnico. Cuando sus dirigentes y los colaboradores inmediatos provienen de la enseñanza superior, tienden a potenciar la actividad de I+D, conceden mas importancia a la formación técnico-científica y sus estrategias tienden a valorizar los saberes adquiridos tratando de iniciar nuevas líneas de negocio. Sin embargo, es un hecho constatable que, en general, las PYMEs suelen emplear pocos titulados superiores. Últimamente se está detectando en algunas de estas empresas un creciente interés por contratar titulados superiores o medios, con una cierta experiencia en labores de investigación, adquirida en Centros de Investigación (Universidades o CSIC) o en Centros de Innovación y Tecnología cuya actividad está íntimamente ligada al sector industrial al que pertenece dicha empresa. La mayoría de ellos se incorporan a los equipos de I+D. Unos pocos pasan a ocupar puestos de dirección que desempeñan con gran eficacia, como consecuencia de su formación anterior. Otros se convierten en empresarios, creando industrias pequeñas y muy tecnificadas sobre la base de su formación científica y su experiencia investigadora. Estas empresas suelen dedicarse a realizar investigación por contrato y a transferir tecnología o a producir bienes de consumo con tecnología muy avanzada. La incorporación de estos técnicos a las empresas constituye, entre otras ventajas, una excelente vía para el intercambio tecnológico y, por tanto, de enriquecimiento para las empresas receptoras.

\section{EVOLUCIÓN DE LA INDUSTRIA DE BALDOSAS CERÁMICAS EN ESPAÑA}

En el año 1999, España e Italia fueron los primeros países productores de baldosas cerámicas, con una fabricación estimada de 602 y 605 millones de $\mathrm{m}^{2}$ respectivamente, con una cuota conjunta en torno al $30 \%$ de la producción mundial, si se excluye China. Este porcentaje se reduce considerablemente si se tienen en cuenta los últimos datos procedentes de China que le atribuyen una producción anual en torno a los 1600 millones de m2, en 1999, de los que solo se exportaron unos 19 millones (figura 1).

El liderazgo de España e Italia viene corroborado por los datos relativos al comercio exterior mundial, esto es, eliminando el consumo interno de cada país fabricante, Italia y España acaparan casi el 70\% del comercio mundial (Italia con una cuota próxima al 40\% y España con alrededor de un 28\%). Concretamente en España se exportó el 45\% de la producción en 1999.

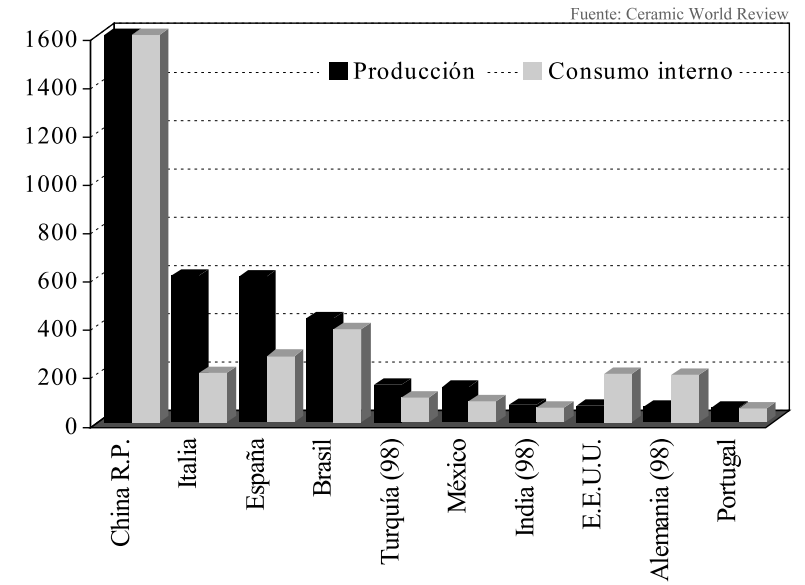

Figura 1. Producción y consumo de los diez primeros países productores (1998-99). 
Un $90 \%$ de las baldosas cerámicas que se fabrican en España están vidriadas. El 10\% restante lo constituyen las baldosas rústicas y las de gres porcelánico (pulido o no) que se suelen utilizar como pavimento. En consecuencia, el consumo de fritas que junto con los pigmentos cerámicos se utilizan para obtener el recubrimiento vidriado de las baldosas esmaltadas, es asimismo un buen indicador de la evolución de la producción de baldosas cerámicas de un país.

También España es líder, actualmente, en la producción de fritas y esmaltes cerámicos, habiendo superado a Italia en la facturación de estas materias primas elaboradas que, en 1999, alcanzó un valor próximo a los ciento once mil millones de pesetas, frente a los noventa y dos mil millones de pesetas de este ultimo país.

El papel preponderante de España, a nivel mundial, en las cifras de producción y ventas de baldosas y de fritas cerámicas, es ya un buen indicador de la magnífica situación que ocupan dichos sectores industriales. No obstante, su extraordinario desarrollo se aprecia mejor si se considera la velocidad de crecimiento que han experimentado estas industrias, en los últimos nueve años (19991-1999).

En efecto, a lo largo de este periodo, la producción de baldosas cerámicas en nuestro país pasó de 228 millones de $\mathrm{m}^{2}$ a 602 millones de $\mathrm{m}^{2}$, casi se triplicó. Pero lo más espectacular fue su evolución respecto a la de la producción italiana, durante el mismo periodo, que pasó de 432 millones de $\mathrm{m}^{2}$ a 605 millones de $\mathrm{m}^{2}$ (figura 2). No solo se igualó la producción de ambos países en 1999, sino que, en nuestro país, prácticamente se duplicó la velocidad de crecimiento experimentada por dicha industria respecto a la de Italia, país que, desde siempre, había sido el primer productor de baldosas cerámicas del mundo.

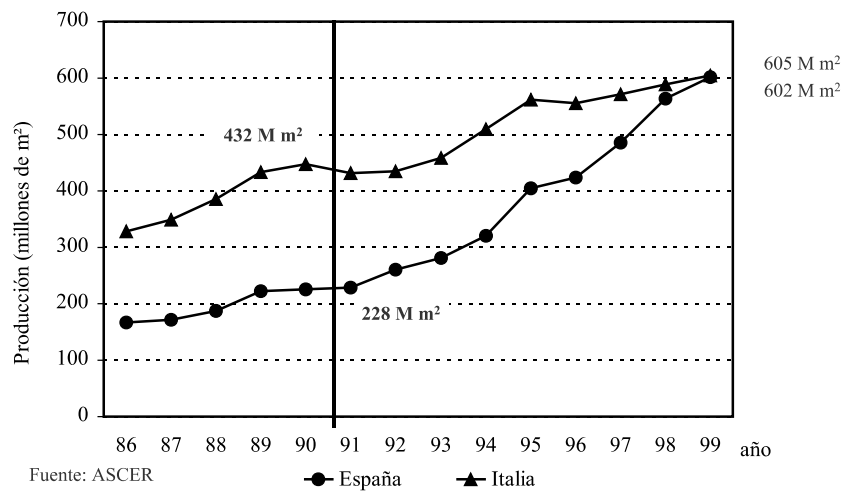

Figura 2. Evolución de la producción de baldosas cerámicas en España e Italia.

Si se evalúa la producción de fritas y esmaltes en función de la facturación, único dato al que se ha tenido acceso, en el periodo 1992-99 evolucionó desde 41200 millones de pts. a 110995 millones de pts. También en esta industria se observa un crecimiento espectacular, durante la última década, respecto a Italia, país con el que se igualó la facturación en 1997 y se superó ampliamente en 1999 (figura 3). Conviene resaltar además que una parte importante de la facturación en Italia correspondió a empresas españolas que exportan a dicho país o que tienen empresas asociadas o filiales en el mismo, lo cual acentúa todavía mas las diferencias a favor de España.

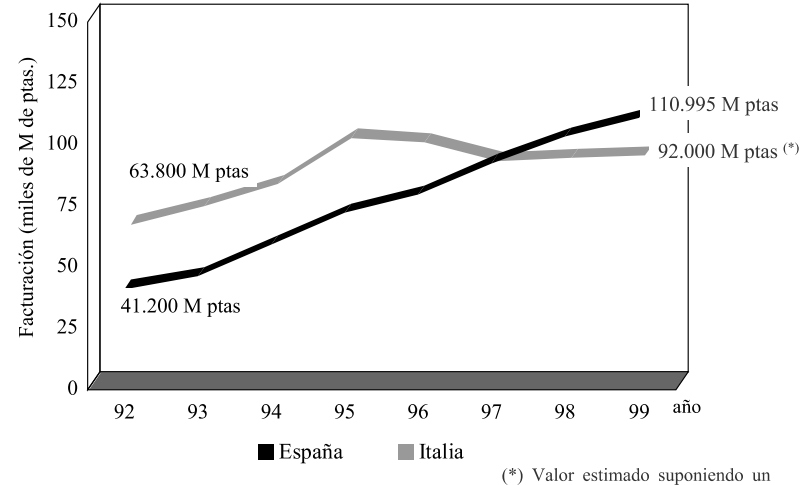

Fuentes: ANFFECC y Ceramic World Review

*) Valor estimado suponiendo

Figura 3. Evolución de la facturación de fritas cerámicas en España e Italia.

\section{EL CLUSTER CERÁMICO DE CASTELLÓN}

Hablar de las industrias de baldosas y de fritas, esmaltes y pigmentos cerámicos en España, equivale a hablar de dicha industria en Castellón. En efecto, el $94 \%$ de la producción de baldosas cerámicas (200 fábricas de un total de 225) y el $95 \%$ de la producción de fritas y pigmentos cerámicos (28 fábricas de un total de 32), están concentradas en un área geográfica restringida, de menos de $300 \mathrm{~km}^{2}$ de extensión, situada en la provincia de Castellón (figuras 4 y 5).

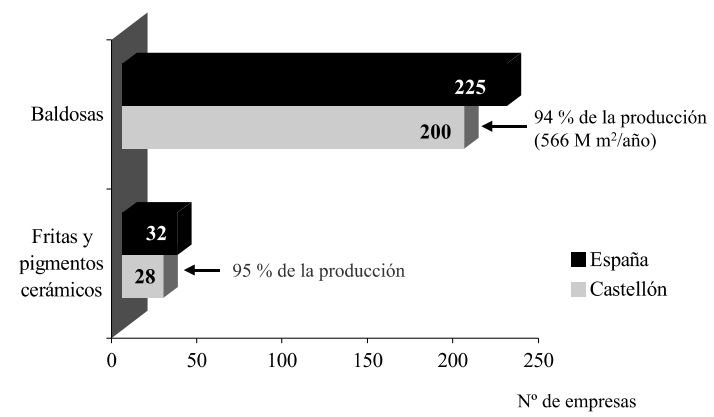

Figura 4. Concentración de las empresas del sector cerámico español.

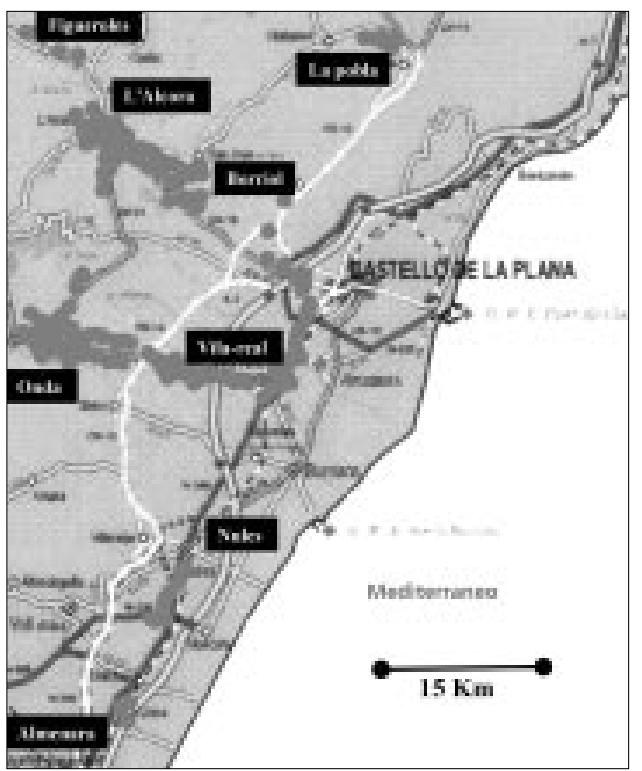

Figura 5. Concentración de las empresas del sector cerámico español. 
Esta agrupación de empresas, por sus características (número de fábricas, tamaño, concentración y potencial económico) constituye lo que en términos económicos se denomina "un sistema productivo local" (cluster).

El empleo directo generado por esta actividad alcanzó en 1999 la cifra de 26000 trabajadores, aunque sólo 26 empresas de baldosas y 6 de fritas y esmaltes tienen más de 200 trabajadores.

Durante el año 1998, ochenta y tres de estas empresas (70 de baldosas cerámicas y 13 de fritas y pigmentos cerámicos) sobrepasaron, individualmente, los 2000 millones de pts. de facturación y once de ellas los 10000 millones de pts.

La mayor parte de las baldosas cerámicas vidriadas se fabrican actualmente en España mediante la técnica de monococción (cocción simultánea del soporte y de la capa de esmalte).

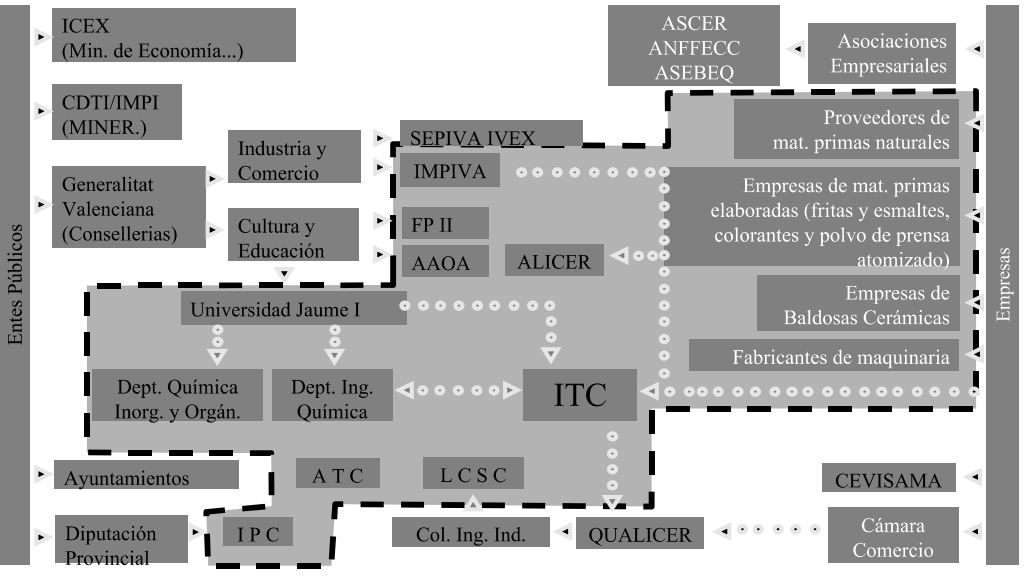

Figura 6. Entidades comprometidas con el proceso de innovación. Durante los últimos quince años, el método de bicocción tradicional (cocción del soporte y del esmalte por separado) ha sido sustituido por el de monococción casi en su totalidad, lo que ha hecho necesario que los fabricantes de fritas hayan hecho un gran esfuerzo para elaborar nuevas fritas adecuadas a las necesidades del proceso de monococción. Este cambio tecnológico, producido en los últimos años, ha supuesto un gran esfuerzo innovador en las empresas de baldosas y de fritas cerámicas.

\section{FACTORES QUE HAN INFLUIDO EN EL DESARROLLO DE ESTE SISTEMA PRODUCTIVO LOCAL (CLUSTER)}

Como se acaba de ver, por las cifras que se han expuesto, tanto la industria de baldosas cerámicas como la de fritas y de pigmentos cerámicos han experimentado, en España, un rápido y extraordinario desarrollo, habiendo mejorado considerablemente su competitividad, sobre todo a lo largo de los últimos nueve años, hasta convertirse en uno de los sectores industriales más dinámicos del País.

Este extraordinario desarrollo, solo repetido a escala mundial en muy contados casos y en otro tipo de industrias muy tecnificadas, no es casual. Se debe a una serie de factores y circunstancias que han concurrido en la reducida área geográfica, antes mencionada, donde está ubicada el $95 \%$ de la producción de las empresas de estos sectores industriales. A continuación se expondrán los factores que han contribuido, de forma decisiva, a tan rápida y favorable evolución.

\subsection{Entramado socio-económico}

La concentración de empresas de baldosas cerámicas y de fritas y pigmentos existente en el área geográfica antes indicada y su fuerte incidencia en la sociedad, ha propiciado el desarrollo de un entramado socio-económico, basado en la colaboración entre los entornos productivo, corporativo e institucional, que ha favorecido la implantación de la innovación tecnológica. Ha sido un proceso interactivo en el que, además de las empresas del sector, vienen participando una serie de Asociaciones Empresariales, Asociaciones Profesionales, Entes de la Administración Pública (de ámbito estatal y local), Instituciones Docentes y de Investigación y un Centro de Innovación y Tecnología, ubicados en Castellón y su provin-

cia. Además, anual o bianualmente, se realiza una serie de eventos (feria monográfica, congresos) relacionados con dichos sectores industriales. La interrelación entre todos ha contribuido a potenciar el crecimiento del sector cerámico y al desarrollo del proceso de innovación en el mismo. Entre estas entidades y organizaciones cabe destacar las siguientes (figura 6):

- ASCER, la Asociación Nacional de Fabricantes de Baldosas Cerámicas.

- ANFFECC, la Asociación Nacional de Fabricantes de Fritas, Esmaltes y Colorantes Cerámicos.

- ASEBEC, la Asociación Española de Maquinaria y Bienes de Equipo para la Industria Cerámica.

- EL Colegio Oficial de Ingenieros Industriales, copatrocinador de QUALICER.

- ATC, la Asociación de Técnicos Cerámicos, elemento aglutinador de los técnicos del sector y órgano difusor de los conocimientos y recursos tecnológicos entre los mismos.

- ALICER, la Asociación para la Promoción del Diseño Cerámico. Forma parte de la red de Institutos Tecnológicos del IMPIVA.

- El Laboratorio Cerámico Sebastián Carpi, del Colegio Oficial de Ingenieros Industriales de Castellón, que es un laboratorio de ensayos de producto acabado.

- El Instituto de Promoción Cerámica, de la Diputación Provincial de Castellón, difusor de los usos arquitectónicos de las baldosas. Actualmente lidera un programa de colocación de las mismas.

- QUALICER (Congreso Mundial del Azulejo y del Pavimento Cerámico), de periodicidad bianual, donde se exponen parte de los logros tecnológicos del sector.

- CEVISAMA, feria monográfica de pavimento y revestimiento cerámicos y afines, que se celebra anualmente.

- La Universitat JAUME I de Castellón.

- El Instituto de Tecnología Cerámica, que es un Centro de Innovación y Tecnología, creado con la misión de apoyar y potenciar la industria cerámica. Forma parte de la red de Institutos Tecnológicos del IMPIVA.

\subsection{Entidades comprometidas con el proceso de innovación}

Aun cuando todas las empresas, entidades y organizaciones que forman este entramado han participado, en mayor o menor grado, en el proceso de innovación desarrollado en los 
sectores industriales de baldosas cerámicas y de fritas, esmaltes y colorantes cerámicos, hay algunas que han participado de forma más destacada. En la figura 6 están ubicadas dentro del entorno constituido por la línea punteada negra. A continuación se resume su contribución a la aportación de los recursos humanos y tecnológicos necesarios para que se haya producido y se siga desarrollando la innovación tecnológica en dichos sectores industriales.

\subsubsection{PREPARACIÓN DE TÉCNICOS CUALIFICADOS (RECURSOS HUMANOS)}

En lo que se refiere a la preparación de técnicos cualificados, con formación específica en cerámica, existe una amplia oferta de enseñanzas, tanto regladas como no regladas.

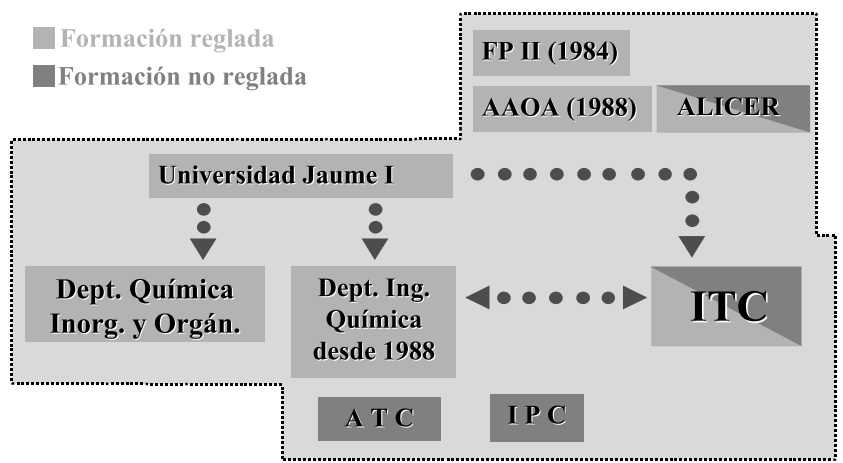

Figura 7. Formación de técnicos cualificados.

La formación reglada en cerámica, a nivel de Formación Profesional de Segundo Grado, se imparte: en la Escuela de Formación Profesional (Especialidad de Cerámica Industrial), desde 1984, y en la Escuela de Artes Aplicadas y Oficios Artísticos (Diseño de revestimientos Cerámicos), desde 1988, ambas ubicadas en Castellón. Asimismo, desde este curso 2000-2001, se imparte en el Instituto de Enseñanza Secundaria de Onda.

La formación técnica reglada, a nivel de segundo y tercer ciclo universitarios, orientada al procesado de materiales cerámicos, se viene impartiendo en el Campus Universitario de Castellón desde 1988, primero en el Colegio Universitario y mas tarde, desde su creación, en la Universitat Jaume I . La titulación de Licenciado en Química (Especialidad de Química Industrial) del Plan de Estudios recientemente extinguido, con un contenido importante en materias de Tecnología Cerámica, se impartió desde octubre de 1988 a junio de 1999. La titulación de Ingeniero Químico , especializado en la industria cerámica, se cursa desde octubre de 1993. Es importante resaltar que en el plan de estudios de Ingeniero Químico, además de las asignaturas troncales propias de la titulación, se han incluido todas las asignaturas de Tecnología Cerámica (con el mismo número de horas semanales) que contiene la titulación de Ingeniería Cerámica (Ceramic Engineering) que se imparte actualmente en el New York State College of Ceramics de la Alfred University (USA), quizás el Centro Superior mas prestigioso del mundo en el área de conocimiento de la Tecnología Cerámica.
La Universitat Jaume I ofrece además las titulaciones de Licenciado en Químicas (con algunas asignaturas de Cerámica) y las de Ingeniero Industrial e Ingeniero Técnico Mecánico, en las que se está empezando a preparar técnicos de grado superior y medio que pueden desempeñar un importante papel en la industria cerámica.

El número de titulados superiores egresados de la Universitat Jaume I, desde 1990, con formación específica en Tecnología Cerámica, ha sido de 315 titulados en Química (Especialidad de Química Industrial) y 167 titulados en Ingeniería Química, en total 482 (figura 8).

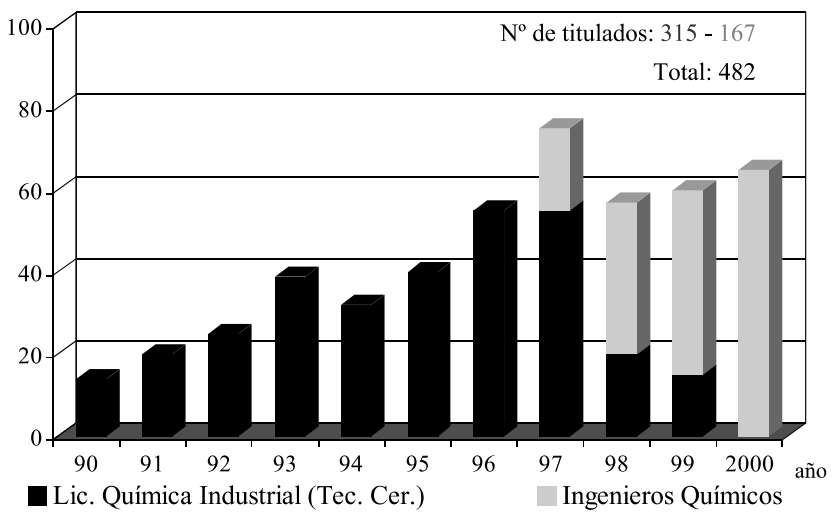

Figura 8. Contribución de la Universidad Jaume I a la formación de técnicos.

En formación no reglada existe, desde hace años, una oferta creciente de cursos de reciclaje y especialización, sobre temas relacionados con la industria cerámica, a diferentes niveles, dirigidos al personal técnico de este sector industrial, promocionados por distintas entidades y organismos, entre los que destacan los auspiciados por el Colegio Oficial de Ingenieros Industriales, por la Asociación de Técnicos Cerámicos y por la Cámara de Comercio e Industria. ALICER viene organizando cursos de formación y reciclaje de diseñadores y el Instituto de Promoción Cerámica desarrolla cursos sobre la problemática de la colocación de baldosas cerámicas. El ITC ha organizado 46 cursos de esta naturaleza, durante los últimos diez años, a los que han asistido 1247 técnicos de grado superior y medio (figuras 9 y 10 ).

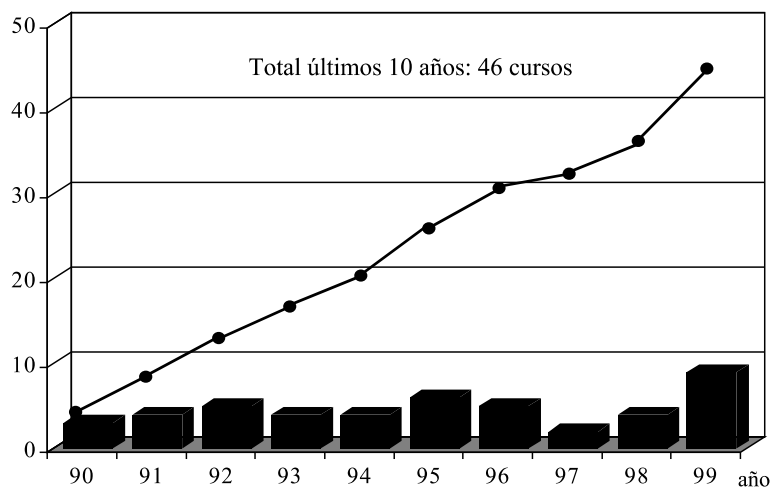

Figura 9. Contribución del ITC: Cursos de reciclaje impartidos a técnicos superiores y medios (no reglada). 


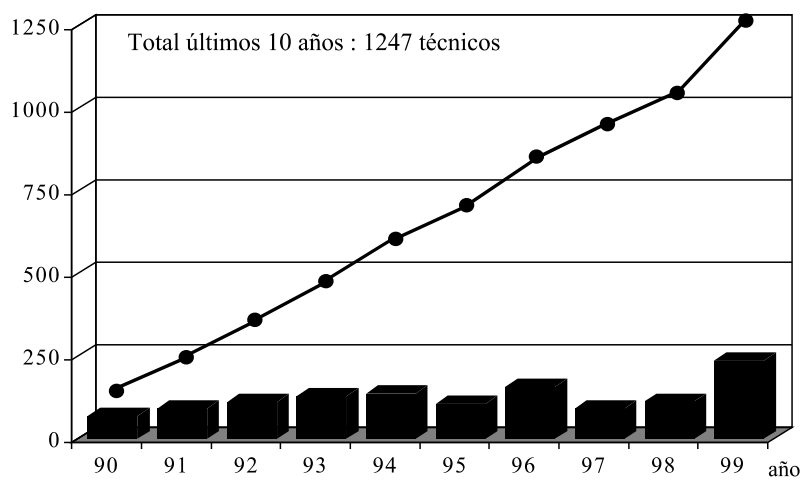

Figura10. Contribución del ITC: Asistencia de técnicos a los cursos de reciclaje.

En el capítulo de formación cabe incluir también la adquirida por los titulados superiores y medios que, después de un periodo de trabajo de uno o varios años, como colaboradores técnicos, en el ITC, son contratados por las empresas de cerámica. Durante los últimos nueve años han sido contratados, por las empresas, 93 técnicos superiores que formaban parte de la plantilla del ITC (figura 11).

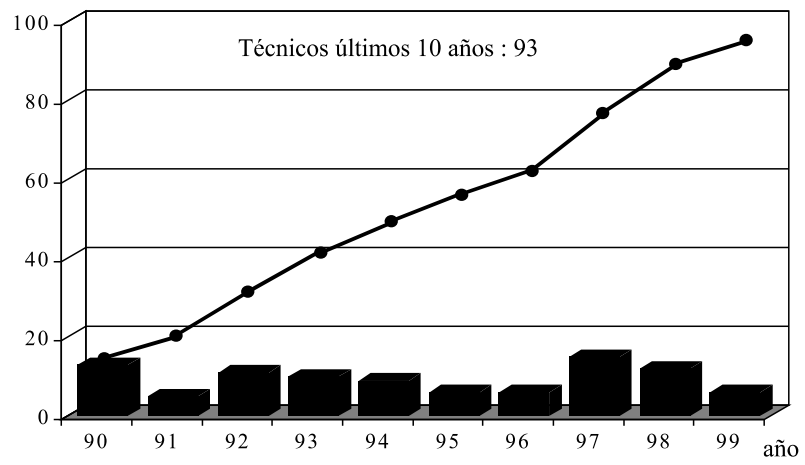

Figura 11. Contribución del ITC: Técnicos superiores y medios contratados por empresas del sector cerámico procedentes de la plantilla del ITC.

Como consecuencia de lo que antecede, el sistema productivo de baldosas cerámicas de Castellón se caracteriza por la alta cualificación de sus recursos humanos. En efecto, según los últimos estudios, un $60 \%$ de los cargos directivos son graduados superiores universitarios, con carreras técnicas o experimentales en su mayoría. El número de técnicos con titulación universitaria que trabaja en el sector se puede estimar en más de 500, en su mayoría ingenieros químicos, químicos, ingenieros industriales e ingenieros mecánicos, esto es, casi el $6 \%$ del número total de trabajadores. En el caso de empresas de fritas y esmaltes, este porcentaje puede llegar a superar incluso el $15 \%$ de los trabajadores.

\subsubsection{RECURSOS TECNOLÓGICOS (FIGURA 12)}

Las empresas de fritas y esmaltes y las de pigmentos cerámicos han contribuido decisivamente al proceso de innovación en el sector cerámico español, tanto por el esfuerzo que han realizado para generar tecnología como para transferirla. Se han preocupado no solo de elaborar nuevas fritas y pig- mentos en el momento oportuno, sino también de transferir la tecnología de su aplicación a las empresas de pavimento y revestimiento cerámicos. Estas empresas suelen diseñar y desarrollar modelos, con las fritas o pigmentos que producen, ofreciendo, a las fábricas de baldosas cerámicas, las materias primas elaboradas y la tecnología necesarias para reproducir industrialmente las piezas, así como la asistencia técnica que se precise, en el caso de que surja algún problema al tratar de fabricarlas.
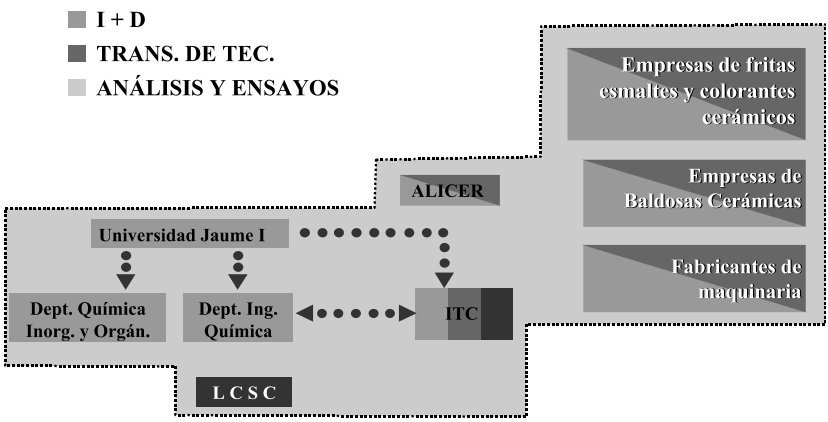

Figura 12. Recursos tecnológicos.

Las empresas de baldosas cerámicas, las de fritas y esmaltes y las de pigmentos cerámicos, han intervenido activamente en el proceso innovador desarrollado en dichos sectores industriales modificando, en muchos casos, el equipo y la maquinaria importados, a fin de adaptar el proceso de fabricación a sus necesidades concretas. En estudios realizados, se ha puesto de manifiesto que un $71 \%$ de las empresas afirma haber participado en el diseño de los equipos adquiridos y un $76 \%$ asegura haber hecho modificaciones en la maquinaria comprada, a sugerencia de su personal técnico.

También han colaborado activamente en el proceso innovador las empresas fabricantes de maquinaria para la industria cerámica que se han venido creando, con gran esfuerzo (debido a la fuerte competencia externa, sobre todo italiana) en nuestro país.

La Asociación para la Promoción del Diseño Industrial Cerámico (ALICER) viene contribuyendo activamente a la innovación tecnológica, en el aspecto del diseño, mediante la realización de proyectos de investigación en diseño y desarrollo de productos cerámicos, desarrollo de nuevos efectos decorativos y de nuevas aplicaciones arquitectónicas de la cerámica, desarrollo de prototipos cerámicos, de procesos decorativos (especialmente serigráficos), etc.

En lo que se refiere a la contribución de Centros de Investigación a la generación de tecnología para los sectores industriales de baldosas cerámicas y de fritas, esmaltes y colorantes cerámicos, se está detectando últimamente cierto interés en participar en algunos proyectos de investigación, en colaboración con empresas, por parte de algún Departamento de la Universidad Politécnica de Valencia hasta hace muy poco, se preocupaban escasamente de la problemática de estos sectores industriales.

Todas las demás actividades de investigación que se realizan, fuera de las empresas, en apoyo de dichos sectores industriales, se concentran en la Universitat JAUME I, desarrollándose en el Departamento de Química Inorgánica y Orgánica y en el Instituto de Tecnología Cerámica.

En el Departamento de Química Inorgánica y Orgánica se vienen realizando, desde hace años (inicialmente en colaboración con un grupo de investigación del Departamento de Química 
Inorgánica de la Universitat de Valencia), trabajos de investigación sobre vidriados y pigmentos cerámicos, algunos de ellos en colaboración con empresas de dicho sector industrial, participando en proyectos subvencionados por la Administración (europea, estatal o de la Comunidad Autónoma).

En el Instituto de Tecnología Cerámica (ITC) se desarrollan todas las actividades que, al principio de esta exposición, se ha indicado era necesario realizar para aportar a las empresas los recursos tecnológicos necesarios para la innovación. Por esa razón está acreditado oficialmente como Centro de Innovación y Tecnología, desde 1998, por la Comisión Interministerial de Ciencia y Tecnología (CICYT).

El ITC es un Instituto Universitario Mixto, actualmente constituido por convenio entre la Universitat Jaume I y la Asociación de Investigación de las Industrias Cerámicas que agrupa, en estos momentos, a 225 empresas del sector. Viene trabajando exclusivamente para las industrias de baldosas cerámicas y de fritas, esmaltes y colorantes cerámicos desde 1975, bajo diversas denominaciones, primero en el seno de la Universitat de Valencia (Estudi general) y luego integrado en la Universitat Jaume I de Castellón (desde su creación), con un bypass fugaz por el Parque Tecnológico de Valencia. Forma parte de la red de Institutos Tecnológicos del IMPIVA (Instituto de la Mediana y Pequeña Empresa de la Generalitat Valenciana).

En lo que a su aportación de recursos tecnológicos se refiere, el ITC desarrolla las siguientes actividades: a) Desarrollo de Proyectos de (I+D) en colaboración con empresas de los sectores industriales que se están considerando (subvencionados parcialmente o no por Organismos de la Administración europea, estatal o de la Comunidad Autónoma); b) transferencia de tecnología y asistencia técnica; c) servicios tecnológicos - análisis y ensayos; control de calidad del producto acabado (el laboratorio está acreditado nacional e internacionalmente); garantía de calidad; e información y documentación.

Como muestra de la labor realizada por el ITC, durante los últimos nueve años (1991-99), en las actividades que se acaban de mencionar, se aportan algunas cifras a continuación. Se han realizado 325 proyectos de (I+D) y de transferencia de tecnología, contratados por las empresas del sector cerámico (figura 13), que han supuesto una facturación de 1487 millones de pts.(figura 14). Muchos de ellos han sido parcialmente subvencionados con fondos públicos, por un montante total de 382 millones de pts. (figura 15). Se han efectuado 88500 análisis y ensayos (figura 16), con unos ingresos totales, por dicho concepto, de 655 millones de pts., lo que supone una media anual que oscila alrededor de los 74 millones de pts. (figura 17).

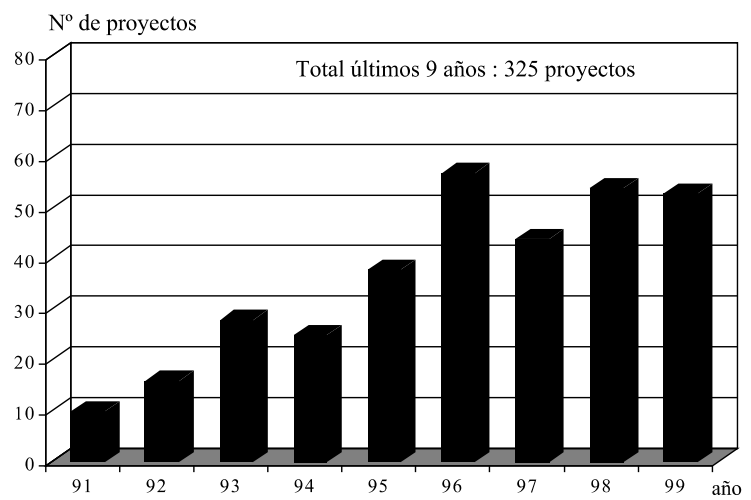

Figura 13. Contribución del ITC: Número de proyectos de I+D (91-99).

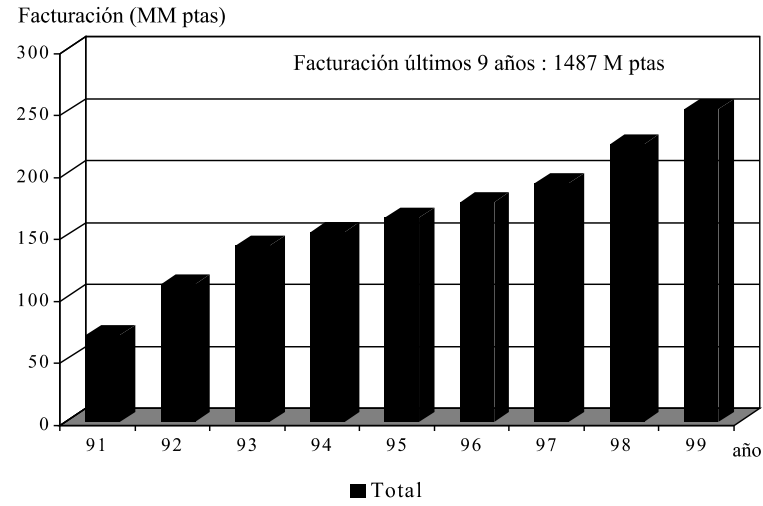

Figura 14. Contribución del ITC: Ingresos por proyectos de I+D (91-99).

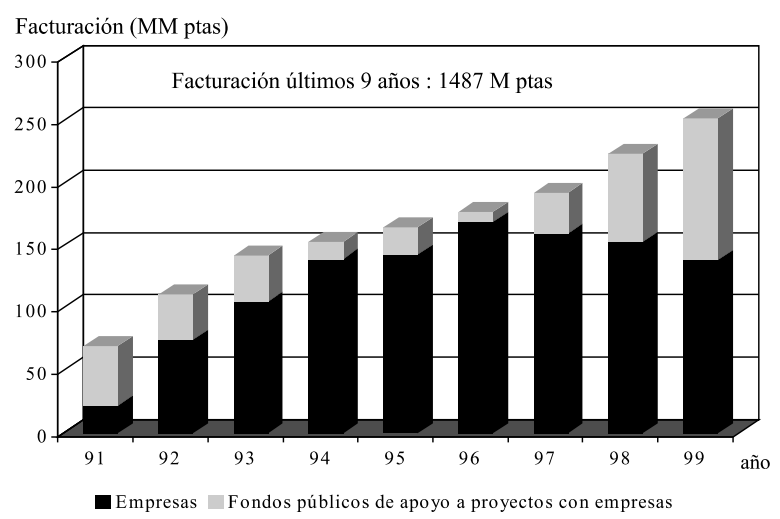

Figura 15. Contribución del ITC: Ingresos por proyectos de I+D (91-99).

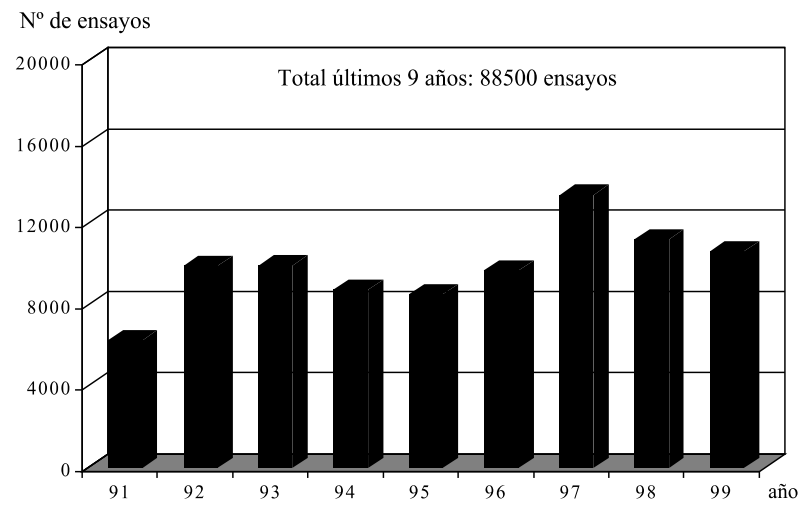

Figura 16. Contribución del ITC: Número de ensayos (91-99).

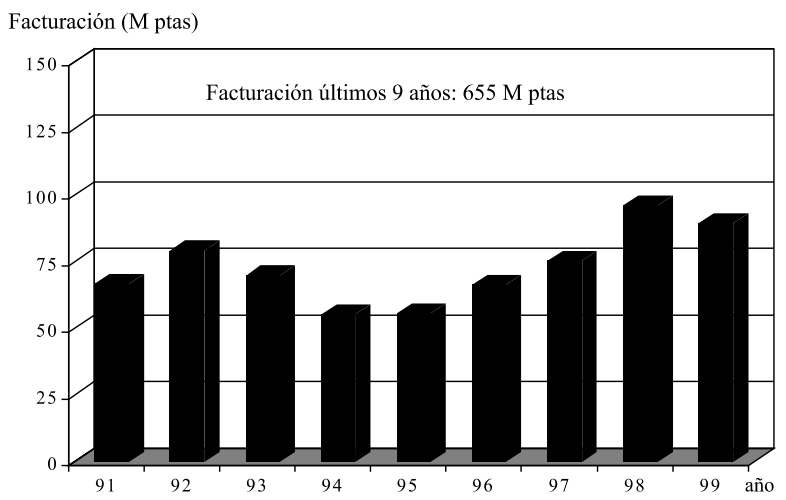

Figura 17. Contribución del ITC: Ingresos por ensayos (91-99). 
Dentro de la labor realizada en el campo de la investigación aplicada cabe mencionar la colaboración y apoyo prestados por el ITC a los congresos de QUALICER, desde sus comienzos (1990). Su aportación de ponencias y posters de investigación ha supuesto, en cada congreso, alrededor del 50\% de los trabajos presentados en la sección G1 (Proceso de Fabricación), una de las dos secciones (la otra es Colocación) en las que se encuadran los trabajos que se presentan a dichos congresos (figura 18).

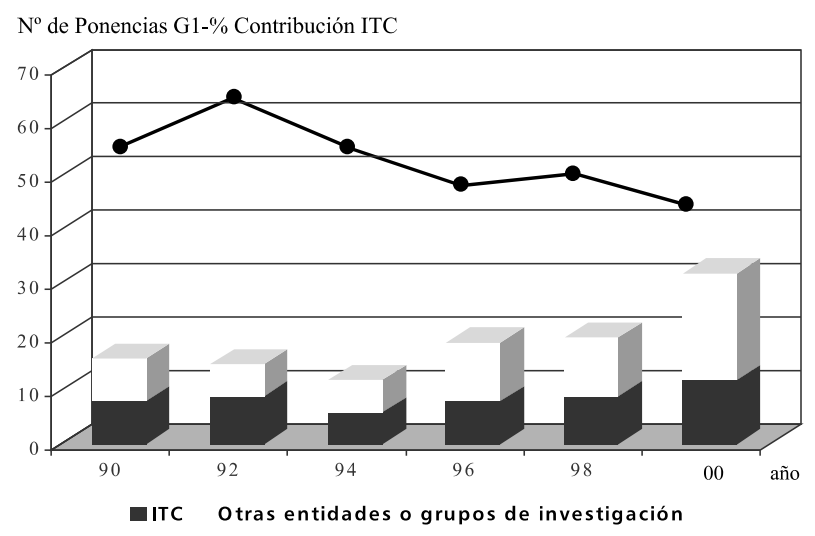

Finalmente, en el capítulo de servicios (análisis y ensayos), cabe citar al Laboratorio Cerámico Sebastián Carpi, perteneciente al Colegio Oficial de Ingenieros Industriales de Castellón, que viene realizando una importante labor de apoyo a la industria cerámica, desde hace 26 años.

En nuestra opinión la interacción de todas las actividades mencionadas que han sido desarrolladas por los entes que forman el entramado antes descrito, ha contribuido decisivamente al proceso innovador en la industria cerámica de Castellón.

Recibido: 08.11 .00

Aceptado: 23.12 .00

Figura 18. Contribución del ITC: Participación en QUALICER

(Ponencias G1).

\section{Seventh Conference and Exhibition of the European Ceramic Society

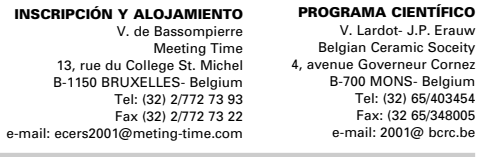

La European Ceramic Society fue constituida en 1987 con los objetivos de coordinar y promover los estudios e investigación sobre cerámica en Europa. Está compuesta por 20 Sociedades Nacionales $y$ otras 8 sociedades nacionales están negociando su incorporación Cada dos años, la ECERS organiza un congreso exposición en uno de los países miembros.

Las conferencias previas han sido organizadas

La VII edición será organizada por la Belgian Ceramic Society y tendrá lugar en Septiembre en el Oud Sint Jan Congrescentrum de Brujas (Belgica)

El tema central serán los materiales cerámicos en su sentido más amplio desde los análisis arqueométricos, la cerámica tradicional, hasta los temas mas candentes de la cerámica avanzada. Esta sección incluirá entre otros temas como: piezas antidesgaste, componentes de alta temperatua, y materiales funcionales con propiedades eléctricas, magnéticas, térmicas y ópticas especiales, para aplicación en áreas como componentes informáticos, comunicación, electrónica, optoeléctrónica, etc.

La conferencia estará dividida en tres grandes sesiones: procesamiento, materiales y sesión general que incluirá métodos de caracterización y normalización. Se dedicará asimismo un sesion especial a la Educación y Formación en Cerámica

a desarrollarán las conferencias invitadas, comunicaciones, carteles y discusiones, de acuerdo con el siguiente esquema preliminar:

\section{PROCESAMIENTO}

- Conformado, Sinterización, Mecanizado, Tecnologías Emergentes

- Unión, Interfases, Recubrimientos

\section{MATERIALES}

- Materiales Cerámicos con aplicaciones en Ingeniería

- Materiales Compuesto

- Biocerámica

- Refractarios

- Cementos

- Cerámica Porosa

MATERIALES CON FUNCIÓN GRADIENTE

- Procesamiento, Aplicaciones en energia, medicina e ingeniería

- Métodos de medida y caracterización

SESIÓN GENERAL

- Caracterización y Normalización

- Medio ambiente y reciclado

CONCURSOS Y PREMIOS

Premio Stuijts

Las sesiones del Congreso incluirán también la Entrega del Premio Stuijts destinado a premiar al investigador, que a juicio del Comité Director de la ECERS y de los organizadores haya hecho a lo largo de su carrera profesional una contribución especial en el campo de la Ciencau Cerámica y haya sido aplicado en beneficio de la Industria Cerámica Europea

\section{Premio a las mejores Comunicaciones y Carteles de Estudiantes} Durante la Conferencia el Comité de Educación de ECERS organizará el Concurso a la
Mejor Comunicación. Cada Sociedad Nacional elegirá el trabajo de un estudiante de su pais. El elegido tendra que presentar su trabajo, en inglés, ante un Jurado Internacional, durate 15 minutos, seguido de una discusión de al menos 5 minutos.

\section{Premio al Mejor Cartel}

Los estudiantes que quieran participar deberán comunicarlo al Comité de Educación de ECERS y deberán indicarlo en el cartel. En este caso no habrá selección previa por las Sociedades Nacionales. El único requisito es que el joven investigador sea el primer do, dato que deberá ser certificado por su supervisor académico

Todos los premios serán entregados durante la cena de Clausura del Congreso FECHAS CLAVE

31-10-2000 Fecha límite envío título y resumen 30-04-2001 Programa Provisional y envío ficha inscripción
01-05-2001 Fecha limite para enviar los trabajos completos

\section{DATOS ENVÍO RESUMEN}

Idioma Ingl

Título. Arial Bold 14 pts, negrilla (Centrado)

Dejar una línea entre titulo y autores

Autor (es) y afiliacion. Arial 14 (Centrado)

Dejar una línea entre autores y resumen

El texto irá en Arial 12 puntos, justificado y con todos los márgenes de $2,5 \mathrm{~cm}$. E

Enviar por correo electrónico a 2001@bcrc be 